\title{
Lyapunov type equation for discrete exponential trichotomies
}

\author{
Davor Dragičević \\ School of Mathematics and Statistics, University of New South Wales, Sydney NSW 2052, Australia. \\ Communicated by D. Baleanu
}

\begin{abstract}
For a nonautonomous dynamics obtained by a sequence of linear operators acting on an arbitrary Hilbert space, we give a complete characterization of the notion of a uniform exponential trichotomy in terms of what can be considered to be a discrete version of the Lyapunov equation. We then use this characterization to study the stability of exponential trichotomies under small linear and nonlinear perturbations. (C)2017 All rights reserved.
\end{abstract}

Keywords: Exponential trichotomies, robustness, perturbations.

2010 MSC: 34D09, 37L45.

\section{Introduction}

The notions of an exponential dichotomy and trichotomy play a central role in the qualitative theory of differential equations and dynamical systems. Indeed, under the assumption that a trajectory admits an exponential dichotomy, one is able to establish the existence of invariant stable and unstable manifolds as well as the nonautonomous version of the Grobman-Hartman theorem. On the other hand, under the assumption that the trajectory admits an exponential trichotomy, one can develop the center manifold theory (see $[8,12,16-19,29,50,51])$. We refer to $[10,15,31,33,49]$ for a detailed discussion, many historical comments and further references. Due to the importance of those notions, it is of considerable interest to obtain their useful characterizations and this is the main theme of the present paper.

More precisely, motivated by the characterization of hyperbolic operators on Hilbert spaces given in [20], we obtain a full characterization of the notion of a uniform exponential trichotomy for a nonautonomous dynamics defined by a sequence of linear operators on an arbitrary Hilbert space. The notion of an exponential trichotomy essentially corresponds to have a splitting of the phase space into three directions, one of which is contracting (with exponential rate) under the action of dynamics, the second is expanding under the action of the dynamics while the third direction (called the center direction) does not have to exhibit either contraction or expansure but one still requires a certain control on the growth along this direction. This notion includes the notion of an exponential dichotomy as a particular case when there is no center direction. We emphasize that the term trichotomy has been used in the literature for various

Email address: d.dragicevic@unsw.edu.au (Davor Dragičević)

doi:10.22436/jnsa.010.04.57

Received 2016-11-10 
different notions. For example, the notion of trichotomy introduced in [25] essentially corresponds to have an exponential dichotomy on both positive and negative line but not necessarily on the whole line (see $[1,26,36]$ for related works). On the other hand, the notion of trichotomy used in the present paper is motivated by the concept of the partial hyperbolicity introduced in [14] which has since then became an important part of the modern smooth ergodic theory (see [32] for a detailed exposition). A similar but weaker (in the sense that it requires less control on the central direction) concept of trichotomy has been extensively studied by Sasu and Sasu [43, 44, 46-48]. For some recent contributions which involve the study of nonuniform trichotomies and trichotomies with not necessarily exponential growth rates, we refer to $[3,5,7-9,11,12,34,35]$.

Our characterization of exponential trichotomies is inspired by the related works which go back to Lyapunov who proved that an $n \times n$ matrix $A$ has all characteristic roots with negative real-parts, if and only if for every positive definite Hermitian matrix $H$, there exists a unique positive definite Hermitian matrix B such that

$$
A^{*} B+B A=-H .
$$

In [20] this theorem was extended to the case of bounded linear operators acting on Hilbert spaces and applied to the asymptotic study of the linear differential equation with constant coefficients of the form $x^{\prime}=A x$ where $A$ is a bounded operator on an arbitrary Hilbert space. The case when $A$ is not necessarily bounded was first considered by Datko [21] and consequently in a series of works [27, 30, 37, 38]. In the present paper we consider:

- the case of discrete time. The principal motivation for this is a general strategy of the discretization of dynamics which involves passing from continuous to discrete time since for many problems, it is easier to threat the case of discrete then continuous time. In the context of nonautonomous dynamics this strategy is outlined in detail for example in [33];

- the case of nonautonomous dynamics. Indeed, autonomous dynamics is not sufficient to threat numerous problems that arise in applications and thus the study of the time-dependent dynamics cannot be avoided. In the context of the present paper, one may consider that our results extend (in various directions) those in [20] which can be applied to the study of a linear difference equation with constant coefficients $x_{n+1}=A x_{n}$ to results which enable us to consider a linear difference equations of the form $x_{n+1}=A_{n} x_{n}$, where now the coefficients are allowed to depend on the time variable $n$;

- the notion of an exponential trichotomy which as already emphasized includes the concepts of stability and dichotomy as very special cases.

We emphasize that some previous works did consider the problem of characterizing exponential behavior for the nonautonomous dynamics in terms of the appropriate Lyapunov equation (see [22,39-41] and references therein), but that our results are the first one that deal with the case of exponential trichotomies. This can be regarded as a principal contribution of the present paper since the notion of an exponential trichotomy is the weakest one under which one is still able to obtain certain information about the qualitative behavior of the dynamics. Regul. Chaotic Dyn., Our strategy can be briefly outlined as follows: we carefully and in a nontrivial way reduce the study of the nonautonomous dynamics to the study of the autonomous dynamics (on a much larger space), apply appropriate results from [20] and return back to our original dynamics. The approach builds on that in $[6,23]$, where we considered only the case of exponential dichotomies and obtained their characterization in terms of Lyapunov functions rather then in terms of the Lyapunov equation.

We also apply our characterization of exponential trichotomy on the study the persistence of trichotomic behavior under sufficiently small linear and nonlinear perturbations. We refer to [3, 12, 24] for related results using different methods.

To summarize, in the present paper we for the first time: 
- characterize trichotomic behavior in terms of the appropriate Lyapunov equation;

- show how this characterization can be used to study the stability of trichotomic behavior under small perturbations.

Finally, we refer to [28] and [52] for some interesting applications of the Lyapunov type techniques in the study of chaos synchronization and discrete fractional maps.

\section{Preliminaries}

\subsection{Characterization of hyperbolic operators}

Let $X=(X,\langle\cdot, \cdot\rangle)$ be a Hilbert space over $\mathbb{R}$ or $\mathbb{C}$ and let $B(X)$ denote the space of all bounded linear operators acting on $X$. The identity operator on $X$ will be denoted by I. For two self-adjoint operators $A, B \in B(X)$ we write $A \geqslant B$, if $\langle A x, x\rangle \geqslant\langle B x, x\rangle$ for each $x \in X$. Furthermore, we say that the operator $A \in B(X)$ is hyperbolic, if the spectrum of $A$ does not intersect the unit circle $S^{1}=\{\lambda:|\lambda|=1\}$.

We will now recall two important theorems concerning hyperbolic operators that will be used in the following sections. Both of those results are taken from [20].

Theorem 2.1. Let $\mathrm{T} \in \mathrm{B}(\mathrm{X})$ be a hyperbolic operator. Then, every self-adjoint operator $\mathrm{W} \in \mathrm{B}(\mathrm{X})$ with the property that there exists a self-adjoint operator $\mathrm{H} \in \mathrm{B}(\mathrm{X})$ and $\delta>0$ such that

$$
\mathrm{T}^{*} \mathrm{WT}-\mathrm{W}=-\mathrm{H} \text { and } \mathrm{H} \geqslant \delta \mathrm{I},
$$

is necessarily invertible.

Theorem 2.2. Let $\mathrm{T} \in \mathrm{B}(\mathrm{X})$ and assume that there exists a self-adjoint invertible operator $\mathrm{W} \in \mathrm{B}(\mathrm{X})$ such that (2.1) holds with some self-adjoint operator $\mathrm{H} \in \mathrm{B}(\mathrm{X})$ and $\delta>0$. Then, $\mathrm{T}$ is hyperbolic, if and only if there exists a self-adjoint operator $\mathrm{H}^{\prime} \in \mathrm{B}(\mathrm{X})$ and $\delta^{\prime}>0$ such that

$$
\mathrm{TW}^{-1} \mathrm{~T}^{*}-\mathrm{W}^{-1}=-\mathrm{H}^{\prime} \text { and } \mathrm{H}^{\prime} \geqslant \delta^{\prime} \mathrm{I} .
$$

\subsection{Exponential trichotomies and dichotomies}

We recall the notions of a (uniform) exponential trichotomy and dichotomy. Let $X$ be as in the previous subsection. Moreover, let $\mathrm{J} \in\left\{\mathbb{Z}_{0}^{+}, \mathbb{Z}_{0}^{-}, \mathbb{Z}\right\}$ be an interval, where

$$
\mathbb{Z}_{0}^{+}=\{n \in \mathbb{Z}: n \geqslant 0\} \quad \text { and } \quad \mathbb{Z}_{0}^{-}=\{n \in \mathbb{Z}: n \leqslant 0\} .
$$

Given a sequence $\left(A_{m}\right)_{m \in J}$ of linear operators in $B(X)$, we define the associated cocycle $\mathcal{A}(n, m)$ by

$$
\mathcal{A}(\mathrm{n}, \mathrm{m})= \begin{cases}A_{n-1} \cdots A_{m} & \text { if } n>m, \\ \operatorname{Id} & \text { if } n=m,\end{cases}
$$

for $n, m \in J, n \geqslant m$. We say that $\left(A_{m}\right)_{m \in J}$ admits an exponential trichotomy, if there exist projections $P_{m}^{i}: X \rightarrow X$ for $i \in\{1,2,3\}$ and $m \in J$ satisfying

$$
P_{m}^{1}+P_{m}^{2}+P_{m}^{3}=I, \quad A_{m} P_{m}^{i}=P_{m+1}^{i} A_{m},
$$

for $m \in \mathbb{Z}$ and $i \in\{1,2,3\}$ such that the operator

$$
A_{m} \mid \operatorname{Im} P_{m}^{i}: \operatorname{Im} P_{m}^{i} \rightarrow \operatorname{Im} P_{m+1}^{i},
$$

is invertible for each $m$ such that $m, m+1 \in J$ and $i \in\{2,3\}$ and there exist constants

$$
D>0, \quad 0 \leqslant a<b, \quad \text { and } \quad 0 \leqslant c<d,
$$


such that

$$
\left\|\mathcal{A}(m, n) P_{n}^{1}\right\| \leqslant D e^{-d(m-n)}, \quad\left\|\mathcal{A}(m, n) P_{n}^{3}\right\| \leqslant D e^{a(m-n)},
$$

for $m, n \in J$ with $m \geqslant n$ and

$$
\left\|\mathcal{A}(m, n) P_{n}^{2}\right\| \leqslant D e^{-b(n-m)}, \quad\left\|\mathcal{A}(m, n) P_{n}^{3}\right\| \leqslant D e^{c(n-m)},
$$

for $\mathrm{m}, \mathrm{n} \in \mathrm{J}$ with $\mathrm{m} \leqslant \mathrm{n}$, where $\mathcal{A}(\mathrm{m}, \mathrm{n})$ in (2.3) denotes

$$
\left(\mathcal{A}(\mathrm{n}, \mathrm{m}) \mid \operatorname{Im} \mathrm{P}_{\mathrm{m}}^{\mathrm{i}}\right)^{-1}: \operatorname{Im} \mathrm{P}_{\mathrm{n}}^{\mathrm{i}} \rightarrow \operatorname{Im} \mathrm{P}_{\mathrm{m}}^{\mathrm{i}}
$$

for $i=2,3$ respectively. Moreover, we say that the sequence $\left(A_{m}\right)_{m \in J}$ admits an exponential dichotomy, if it admits an exponential trichotomy with $\mathrm{P}_{\mathrm{m}}^{3}=0$ for $\mathrm{m} \in \mathbb{Z}$.

It turns out that in the particular case of dichotomies on $\mathbb{Z}$ the projections are uniquely determined (see $[2,42]$ for example).

Proposition 2.3. Assume that the sequence $\left(A_{m}\right)_{m \in \mathbb{Z}}$ admits an exponential dichotomy. Then,

$$
\operatorname{Im} P_{n}^{1}=\left\{x \in X: \sup _{m \geqslant n}\|\mathcal{A}(m, n) x\|<+\infty\right\} .
$$

Furthermore, Im $P_{n}^{2}$ consists of all $x \in X$ for which there exists a sequence $\left(x_{m}\right)_{m \leqslant n} \subset X$ such that

$$
\sup _{m \leqslant n}\left\|x_{m}\right\|<+\infty \text { and } x_{m}=\mathcal{A}(m, k) x_{k} \text { for } k \leqslant m \leqslant n .
$$

Let

$$
l^{2}=\left\{x=\left(x_{n}\right)_{n \in \mathbb{Z}} \subset X: \sum_{n=-\infty}^{\infty}\left\|x_{n}\right\|<+\infty\right\} .
$$

It is straightforward to verify that $l^{2}$ is a Hilbert space with respect to the scalar product

$$
\langle\mathbf{x}, \mathbf{y}\rangle=\sum_{n \in \mathbb{Z}}\left\langle x_{n}, y_{n}\right\rangle, \quad \mathbf{x}=\left(x_{n}\right)_{n \in \mathbb{Z}}, \quad \mathbf{y}=\left(y_{n}\right)_{n \in \mathbb{Z}} \in l^{2}
$$

We will need the following classical result.

Theorem 2.4. Let $\left(A_{m}\right)_{m \in \mathbb{Z}}$ be a sequence of bounded operators on $X$. The following statements are equivalent:

1. the sequence $\left(A_{m}\right)_{m \in \mathbb{Z}}$ admits an exponential dichotomy;

2. for each $\mathbf{y}=\left(y_{n}\right)_{n \in \mathbb{Z}} \in l^{2}$ there exists a unique $\mathbf{x}=\left(x_{n}\right)_{n \in \mathbb{Z}} \in l^{2}$ such that

$$
x_{n+1}-A_{n} x_{n}=y_{n+1}, \quad \forall n \in \mathbb{Z} .
$$

\section{Characterization of exponential trichotomies on $\mathbb{Z}$}

Theorem 3.1. Assume that the sequence $\left(A_{m}\right)_{m \in \mathbb{Z}} \subset \mathrm{B}(\mathrm{X})$ admits an exponential trichotomy and that there exists $\mathrm{C}>0$ such that

$$
\left\|A_{m}\right\| \leqslant C, \quad \text { for } m \in \mathbb{Z} \text {. }
$$

Then, there exist sequences $\left(S_{m}^{i}\right)_{m \in \mathbb{Z}}, i=1,2$ of bounded, self-adjoint and invertible operators on $X$, sequences

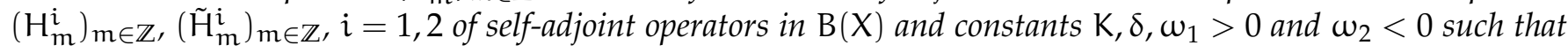
the following inequalities hold for each $\mathrm{m} \in \mathbb{Z}$ and $i \in\{1,2\}$ :

1.

$$
\left\|S_{m}^{i}\right\| \leqslant K \text { and }\left\|\left(S_{m}^{i}\right)^{-1}\right\| \leqslant K
$$


2.

$$
e^{2 \omega_{i}} A_{m}^{*} S_{m+1}^{i} A_{m}-S_{m}^{i}=-H_{m}^{i} \text { and } H_{m}^{i} \geqslant \delta I
$$

3.

$$
e^{2 \omega_{i}} A_{m-1}\left(S_{m-1}^{i}\right)^{-1} A_{m-1}^{*}-\left(S_{m}^{i}\right)^{-1}=-\tilde{H}_{m}^{i}, \quad \text { and } \quad \tilde{H}_{m}^{i} \geqslant \delta I .
$$

Proof. Take $\omega_{1} \in(c, d)$ and consider the sequence $B_{m}=e^{\omega_{1}} A_{m}$. The cocycle associated to the sequence $\left(B_{m}\right)_{m \in \mathbb{Z}}$ is given by

$$
\mathcal{B}(\mathrm{m}, \mathrm{n})=\mathrm{e}^{\mathrm{\omega}_{1}(\mathrm{~m}-\mathrm{n})} \mathcal{A}(\mathrm{m}, \mathrm{n}) .
$$

It follows from (2.2) and (2.3) that

$$
\left\|\mathcal{B}(m, n) P_{n}^{1}\right\| \leqslant D e^{-\left(d-\omega_{1}\right)(m-n)},
$$

for $m \geqslant n$ and that

$$
\left\|\mathcal{B}(m, n) P_{n}^{2}\right\| \leqslant D e^{-\left(b+\omega_{1}\right)(n-m)}
$$

and

$$
\left\|\mathcal{B}(m, n) P_{n}^{3}\right\| \leqslant D e^{-\left(\omega_{1}-c\right)(n-m)},
$$

for $m \leqslant n$. By (3.6) and (3.7), we have

$$
\left\|\mathcal{B}(m, n)\left(P_{n}^{2}+P_{n}^{3}\right)\right\| \leqslant 2 D e^{-\min \left\{b+\omega_{1}, \omega_{1}-c\right\}(n-m)}, \quad \text { for } m \leqslant n .
$$

Let

$$
\lambda=\min \left\{d-\omega_{1}, b+\omega_{1}, \omega_{1}-c\right\}>0 .
$$

It follows from (3.5) and (3.8) that

$$
\left\|\mathcal{B}(m, n) P_{n}^{1}\right\| \leqslant D e^{-\lambda(m-n)}, \quad \text { for } m \geqslant n,
$$

and

$$
\left\|\mathcal{B}(m, n)\left(P_{n}^{2}+P_{n}^{3}\right)\right\| \leqslant 2 D e^{-\lambda(n-m)} \quad \text { for } m \leqslant n .
$$

Choose an arbitrary $\rho \in(0, \lambda)$ and set

$$
\begin{aligned}
S_{m}^{1}= & \sum_{k \geqslant m}\left(\mathcal{B}(k, m) P_{m}^{1}\right)^{*} \mathcal{B}(k, m) P_{m}^{1} e^{2(\lambda-\rho)(k-m)} \\
& -\sum_{k<m}\left(\mathcal{B}(k, m)\left(I-P_{m}^{1}\right)\right)^{*} \mathcal{B}(k, m)\left(I-P_{m}^{1}\right) e^{2(\lambda-\rho)(m-k)} .
\end{aligned}
$$

It follows from (3.9) and (3.10) that

$$
\begin{aligned}
\left|\left\langle S_{m}^{1} x, x\right\rangle\right| \leqslant & \sum_{k \geqslant m}\left\|\mathcal{B}(k, m) P_{m}^{1} x\right\|^{2} e^{2(\lambda-\rho)(k-m)} \\
& +\sum_{k<m}\left\|\mathcal{B}(k, m)\left(I-P_{m}^{1}\right) x\right\|^{2} e^{2(\lambda-\rho)(m-k)} \\
\leqslant & \sum_{k \geqslant m} D^{2} e^{-2 \lambda(k-m)} e^{2(\lambda-\rho)(k-m)}\|x\|^{2} \\
& +\sum_{k<m} 4 D^{2} e^{-2 \lambda(m-k)} e^{2(\lambda-\rho)(m-k)}\|x\|^{2} \\
= & D^{2}\left(\sum_{k \geqslant m} e^{-2 \rho(k-m)}+4 \sum_{k<m} e^{-2 \rho(m-k)}\right)\|x\|^{2} \\
= & K\|x\|^{2},
\end{aligned}
$$


for each $m \in \mathbb{Z}$, where

$$
\mathrm{K}=\mathrm{D}^{2}\left(\sum_{\mathrm{k} \geqslant \mathrm{m}} \mathrm{e}^{-2 \rho(\mathrm{k}-\mathrm{m})}+4 \sum_{\mathrm{k}<\mathrm{m}} e^{-2 \rho(\mathrm{m}-\mathrm{k})}\right)<+\infty .
$$

Obviously, $S_{m}^{1}$ is self-adjoint and thus

$$
\left\|S_{m}^{1}\right\|=\sup _{\|x\|=1}\left|\left\langle S_{m}^{1} x, x\right\rangle\right| \leqslant K, \quad \forall m \in \mathbb{Z} .
$$

Hence, we have obtained the first inequality in (3.2) for $i=1$. Furthermore, we have

$$
\begin{aligned}
& B_{m}^{*} S_{m+1}^{1} B_{m}=B_{m}^{*} \sum_{k \geqslant m+1}\left(\mathcal{B}(k, m+1) P_{m+1}^{1}\right)^{*} \mathcal{B}(k, m+1) P_{m+1}^{1} e^{2(\lambda-\rho)(k-m-1)} B_{m} \\
& -B_{m}^{*} \sum_{k<m+1}\left(\mathcal{B}(k, m+1)\left(I-P_{m+1}^{1}\right)\right)^{*} \mathcal{B}(k, m+1)\left(I-P_{m+1}^{1}\right) e^{2(\lambda-\rho)(m+1-k)} B_{m} \\
& =\sum_{k \geqslant m+1}\left(\mathcal{B}(k, m+1) P_{m+1}^{1} B_{m}\right)^{*} \mathcal{B}(k, m+1) P_{m+1}^{1} B_{m} e^{2(\lambda-\rho)(k-m-1)} \\
& -\sum_{k<m+1}\left(\mathcal{B}(k, m+1)\left(I-P_{m+1}^{1}\right) B_{m}\right)^{*} \mathcal{B}(k, m+1)\left(I-P_{m+1}^{1}\right) B_{m} e^{2(\lambda-\rho)(m+1-k)} \\
& =\sum_{k \geqslant m+1}\left(\mathcal{B}(k, m+1) B_{m} P_{m}^{1}\right)^{*} \mathcal{B}(k, m+1) B_{m} P_{m}^{1} e^{2(\lambda-\rho)(k-m-1)} \\
& -\sum_{k<m+1}\left(\mathcal{B}(k, m+1) B_{m}\left(I-P_{m}^{1}\right)\right)^{*} \mathcal{B}(k, m+1) B_{m}\left(I-P_{m}^{1}\right) e^{2(\lambda-\rho)(m+1-k)} \\
& =\sum_{k \geqslant m+1}\left(\mathcal{B}(k, m) P_{m}^{1}\right)^{*} \mathcal{B}(k, m) P_{m}^{1} e^{2(\lambda-\rho)(k-m-1)} \\
& -\sum_{k<m+1}\left(\mathcal{B}(k, m)\left(I-P_{m}^{1}\right)\right)^{*} \mathcal{B}(k, m)\left(I-P_{m}^{1}\right) e^{2(\lambda-\rho)(m+1-k)} \\
& =e^{-2(\lambda-\rho)} \sum_{k \geqslant m+1}\left(\mathcal{B}(k, m) P_{m}^{1}\right)^{*} \mathcal{B}(k, m) P_{m}^{1} e^{2(\lambda-\rho)(k-m)} \\
& -e^{2(\lambda-\rho)} \sum_{k<m+1}\left(\mathcal{B}(k, m)\left(I-P_{m}^{1}\right)\right)^{*} \mathcal{B}(k, m)\left(I-P_{m}^{1}\right) e^{2(\lambda-\rho)(m-k)} \\
& =e^{-2(\lambda-\rho)} \sum_{k \geqslant m}\left(\mathcal{B}(k, m) P_{m}^{1}\right)^{*} \mathcal{B}(k, m) P_{m}^{1} e^{2(\lambda-\rho)(k-m)}-e^{-2(\lambda-\rho)}\left(P_{m}^{1}\right)^{*} P_{m}^{1} \\
& -e^{2(\lambda-\rho)} \sum_{k<m}\left(\mathcal{B}(k, m)\left(I-P_{m}^{1}\right)\right)^{*} \mathcal{B}(k, m)\left(I-P_{m}^{1}\right) e^{2(\lambda-\rho)(m-k)} \\
& -e^{2(\lambda-\rho)}\left(I-P_{m}^{1}\right)^{*}\left(I-P_{m}^{1}\right) \text {. }
\end{aligned}
$$

Therefore,

$$
\begin{aligned}
B_{m}^{*} S_{m+1}^{1} B_{m}-S_{m}^{1}= & \left(e^{-2(\lambda-\rho)}-1\right) \sum_{k \geqslant m}\left(\mathcal{B}(k, m) P_{m}^{1}\right)^{*} \mathcal{B}(k, m) P_{m}^{1} e^{2(\lambda-\rho)(k-m)} \\
& +\left(1-e^{2(\lambda-\rho)}\right) \sum_{k<m}\left(\mathcal{B}(k, m)\left(I-P_{m}^{1}\right)\right)^{*} \mathcal{B}(k, m)\left(I-P_{m}^{1}\right) e^{2(\lambda-\rho)(m-k)} \\
& -e^{-2(\lambda-\rho)}\left(P_{m}^{1}\right)^{*} P_{m}^{1}-e^{2(\lambda-\rho)}\left(I-P_{m}^{1}\right)^{*}\left(I-P_{m}^{1}\right) .
\end{aligned}
$$

Since $e^{-2(\lambda-\rho)}-1<0$ and $1-e^{2(\lambda-\rho)}<0$, we obtain that

$$
\begin{aligned}
B_{m}^{*} S_{m+1}^{1} B_{m}-S_{m}^{1} & \leqslant-e^{-2(\lambda-\rho)}\left(P_{m}^{1}\right)^{*} P_{m}^{1}-e^{2(\lambda-\rho)}\left(I-P_{m}^{1}\right)^{*}\left(I-P_{m}^{1}\right) \\
& \leqslant-e^{-2(\lambda-\rho)}\left(\left(P_{m}^{1}\right)^{*} P_{m}^{1}+\left(I-P_{m}^{1}\right)^{*}\left(I-P_{m}^{1}\right)\right) .
\end{aligned}
$$


Furthermore, we have

$$
\begin{aligned}
2\left\langle\left(\left(P_{m}^{1}\right)^{*} P_{m}^{1}+\left(I-P_{m}^{1}\right)^{*}\left(I-P_{m}^{1}\right)\right) x, x\right\rangle & =2\left\|P_{m}^{1} x\right\|^{2}+2\left\|\left(I-P_{m}^{1}\right) x\right\|^{2} \\
& \geqslant\left\|P_{m}^{1} x\right\|^{2}+2\left\|P_{m}^{1} x\right\| \cdot\left\|\left(I-P_{m}^{1}\right) x\right\|+\left\|\left(I-P_{m}^{1}\right) x\right\|^{2} \\
& =\left(\left\|P_{m}^{1} x\right\|+\left\|\left(I-P_{m}^{1}\right) x\right\|\right)^{2} \\
& \geqslant\|x\|^{2}
\end{aligned}
$$

for each $x \in X$ which implies that

$$
-e^{-2(\lambda-\rho)}\left(\left(P_{m}^{1}\right)^{*} P_{m}^{1}+\left(I-P_{m}^{1}\right)^{*}\left(I-P_{m}^{1}\right)\right) \leqslant-\frac{1}{2} e^{-2(\lambda-\rho)} I .
$$

Consequently,

$$
\mathrm{B}_{\mathrm{m}}^{*} \mathrm{~S}_{\mathrm{m}+1}^{1} \mathrm{~B}_{\mathrm{m}}-\mathrm{S}_{\mathrm{m}}^{1} \leqslant-\frac{1}{2} e^{-2(\lambda-\rho)} \mathrm{I}, \quad \forall \mathrm{m} \in \mathbb{Z},
$$

and we conclude that (3.3) holds for $i=1$ with

$$
H_{m}^{1}=S_{m}^{1}-e^{2 \omega_{1}} A_{m}^{*} S_{m+1}^{1} A_{m}, \quad \text { and } \quad \delta=\frac{1}{2} e^{-2(\lambda-\rho)}>0 .
$$

We now wish to establish (3.4) for $i=1$. Let us define the operator $T: l^{2} \rightarrow l^{2}$ by

$$
(T \mathbf{x})_{n}=B_{n-1} x_{n-1}, \quad \text { for } \mathbf{x}=\left(x_{n}\right)_{n \in \mathbb{Z}} \in l^{2} .
$$

It follows readily from (3.1) that $\mathrm{T}$ is well-defined and bounded linear operator.

Lemma 3.2. $\mathrm{T}^{*}: \mathrm{l}^{2} \rightarrow \mathrm{l}^{2}$ is given by

$$
\left(T^{*} \mathbf{x}\right)_{n}=B_{n}^{*} x_{n+1}, \quad \forall n \in \mathbb{Z}, \text { and } \mathbf{x}=\left(x_{n}\right)_{n \in \mathbb{Z}} \in l^{2} .
$$

Proof. We define a linear operator $\mathrm{G}: \mathrm{l}^{2} \rightarrow \mathrm{l}^{2}$ by

$$
(G x)_{n}=B_{n}^{*} x_{n+1}, \quad \forall n \in \mathbb{Z}, \quad \text { and } \quad x=\left(x_{n}\right)_{n \in \mathbb{Z}} \in l^{2} .
$$

Then, for every $\mathbf{x}=\left(x_{\mathfrak{n}}\right)_{\mathfrak{n} \in \mathbb{Z}}$ and $\mathbf{y}=\left(y_{n}\right)_{\mathfrak{n} \in \mathbb{Z}} \in l^{2}$ we have that

$$
\begin{aligned}
\langle G \mathbf{x}, \mathbf{y}\rangle & =\sum_{n \in \mathbb{Z}}\left\langle(G \mathbf{x})_{n}, y_{n}\right\rangle \\
& =\sum_{n \in \mathbb{Z}}\left\langle B_{n}^{*} x_{n+1}, y_{n}\right\rangle \\
& =\sum_{n \in \mathbb{Z}}\left\langle x_{n+1}, B_{n} y_{n}\right\rangle \\
& =\sum_{n \in \mathbb{Z}}\left\langle x_{n+1},(T y)_{n+1}\right\rangle \\
& =\langle\mathbf{x}, T \mathbf{y}\rangle,
\end{aligned}
$$

which implies that $\mathrm{G}=\mathrm{T}^{*}$.

It follows from (3.9) and (3.10) that the sequence $\left(B_{n}\right)_{n \in \mathbb{Z}}$ admits an exponential dichotomy. Hence, by Theorem 2.4 we have that the operator I $-\mathrm{T}$ is invertible. However, we can also obtain the following stronger conclusion.

Lemma 3.3. The operator $\mathrm{T}$ is hyperbolic. 
Proof. Take $\lambda \in \mathbb{C}$ such that $|\lambda|=1$. Since the sequence $\left(B_{n}\right)_{n \in \mathbb{Z}}$ admits an exponential dichotomy we conclude that the sequence $\left(\frac{1}{\lambda} B_{n}\right)_{n \in \mathbb{Z}}$ also admits a uniform exponential dichotomy. Hence, it follows from Theorem 2.4 that the operator

$$
\mathbf{x}=\left(x_{n}\right)_{n \in \mathbb{Z}} \mapsto\left(x_{n}-\frac{1}{\lambda} B_{n-1} x_{n-1}\right)_{n \in \mathbb{Z}},
$$

is an invertible linear operator on $l^{2}$. Consequently, the operator

$$
\mathbf{x}=\left(x_{\mathfrak{n}}\right)_{\mathfrak{n} \in \mathbb{Z}} \mapsto\left(\lambda x_{n}-B_{n-1} x_{n-1}\right)_{n \in \mathbb{Z}}
$$

is also invertible on $l^{2}$ and thus $\lambda \notin \sigma(T)$. We conclude that

$$
\sigma(T) \cap S^{1}=\emptyset,
$$

and therefore $\mathrm{T}$ is hyperbolic.

Moreover, we define an operator $W: l^{2} \rightarrow l^{2}$ by

$$
(\mathbf{W} \mathbf{x})_{n}=S_{n}^{1} x_{n}, n \in \mathbb{Z}, \quad \mathbf{x}=\left(x_{n}\right)_{n \in \mathbb{Z}} \in l^{2} .
$$

It follows from the already established first inequality in (3.2) for $i=1$ that $W$ is well-defined. Furthermore, by (3.12) and Lemma 3.2 we have that

$$
\mathrm{T}^{*} \mathrm{WT}-\mathrm{W}=-\mathrm{H}, \quad \text { and } \mathrm{H} \geqslant \delta \mathrm{I},
$$

where I now denotes the identity operator on $\mathrm{l}^{2}$ and where $\mathrm{H}$ is a self-adjoint operator on $\mathrm{l}^{2}$ given by

$$
(H \mathbf{x})_{m}=H_{m}^{1} x_{m}, \quad \mathbf{x}=\left(x_{m}\right)_{m \in \mathbb{Z}} \in l^{2} .
$$

By Theorem 2.1, $\mathrm{W}$ is invertible. Finally, we have the following lemma.

Lemma 3.4. The operator $S_{m}^{1}$ is invertible for all $m \in \mathbb{Z}$ and there exists $\mathrm{K}>0$ such that the second inequality in (3.2) holds for $i=1$.

Proof. We first establish invertibility of operators $S_{m}^{1}$. Suppose that $S_{m}^{1} v=0$ for some $v \in X$. Define $\mathbf{x}=\left(x_{n}\right)_{n \in \mathbb{Z}} \in l^{2}$ by $x_{m}=v$ and $x_{n}=0$ for all $n \neq m$. Then, $W \mathbf{x}=0$ and using invertibility of $W$ we conclude that $\mathbf{x}=0$. Hence, $v=0$ and $S_{\mathrm{m}}^{1}$ is injective for each $\mathrm{m} \in \mathbb{Z}$.

Now take $v \in X$ and define $\mathbf{y}=\left(y_{n}\right)_{n \in \mathbb{Z}} \in l^{2}$ by $y_{m}=v$ and $y_{n}=0$ for all $n \neq m$. Since $W$ is invertible, there exists $\mathbf{x} \in l^{2}$ such that $W \mathbf{x}=\mathbf{y}$. Thus, $(\mathbf{W} \mathbf{x})_{m}=y_{m}$, whence $S_{m}^{1} x_{m}=y_{m}=v$ and $S_{m}^{1}$ is surjective.

Moreover, using the notation from the previous step, we have that

$$
\left(S_{\mathrm{m}}^{1}\right)^{-1} v=\left(\mathrm{W}^{-1} \mathbf{y}\right)_{\mathrm{m}},
$$

and therefore

$$
\left\|\left(S_{m}^{1}\right)^{-1} v\right\|=\left\|\left(W^{-1} \mathbf{y}\right)_{m}\right\| \leqslant\left\|W^{-1} \mathbf{y}\right\| \leqslant\left\|W^{-1}\right\| \cdot\|\mathbf{y}\|=\left\|W^{-1}\right\| \cdot\|v\| .
$$

Hence, $\left\|\left(S_{m}^{1}\right)^{-1}\right\| \leqslant\left\|W^{-1}\right\|$ for all $m \in \mathbb{Z}$ and the proof of the lemma is complete.

On the other hand, using Theorem 2.2 and (3.15), we conclude that there exists $\delta^{\prime}>0$ and a self-adjoint operator $\tilde{H}$ on $l^{2}$ such that

$$
\mathrm{TW}^{-1} \mathrm{~T}^{*}-\mathrm{W}^{-1}=-\tilde{\mathrm{H}} \text {, and } \tilde{\mathrm{H}} \geqslant \delta^{\prime} \mathrm{I},
$$

which easily implies that (3.4) holds for $i=1$ with $\tilde{H}_{m}^{1} v=(\tilde{H} \mathbf{v})_{m}$ where $\mathbf{v}=\left(v_{n}\right)_{n \in \mathbb{Z}}, v_{m}=v$ and $v_{n}=0$ for $n \neq m$. 
We now construct operators $S_{m}^{2}, m \in \mathbb{Z}$. Take $\omega_{2} \in(-b,-a)$ and consider the sequence $C_{m}=e^{\omega_{2}} A_{m}$. The associated cocycle is given by

$$
\mathcal{C}(\mathrm{m}, \mathrm{n})=\mathrm{e}^{\omega_{2}(\mathrm{~m}-\mathrm{n})} \mathcal{A}(\mathrm{m}, \mathrm{n}) .
$$

It follows from (2.2) and (2.3) that

$$
\left\|\mathcal{C}(m, n) P_{n}^{1}\right\| \leqslant D e^{-\left(d-\omega_{2}\right)(m-n)}
$$

and

$$
\left\|\mathcal{C}(m, n) P_{n}^{3}\right\| \leqslant D e^{-\left(-a-\omega_{2}\right)(m-n)}
$$

for $m \geqslant n$ and that

$$
\left\|\mathcal{C}(m, n) P_{n}^{2}\right\| \leqslant D e^{-\left(b+w_{2}\right)(n-m)},
$$

for $m \leqslant n$. By (3.16) and (3.17) we have that

$$
\left\|\mathcal{C}(m, n)\left(P_{n}^{1}+P_{n}^{3}\right)\right\| \leqslant 2 D e^{-\min \left\{d-\omega_{2},-a-\omega_{2}\right\}(m-n)}, \quad \text { for } m \geqslant n .
$$

Let

$$
\lambda^{\prime}=\min \left\{d-\omega_{2},-a-\omega_{2}, b+\omega_{2}\right\}>0 .
$$

It follows from (3.18) and (3.19) that

$$
\left\|\mathcal{C}(m, n)\left(P_{n}^{1}+P_{n}^{3}\right)\right\| \leqslant 2 D e^{-\lambda^{\prime}(m-n)}, \quad \text { for } m \geqslant n,
$$

and

$$
\left\|\mathcal{C}(m, n) P_{n}^{2}\right\| \leqslant D e^{-\lambda^{\prime}(n-m)}, \quad \text { for } m \leqslant n .
$$

Choose an arbitrary $\rho^{\prime} \in\left(0, \lambda^{\prime}\right)$ and set

$$
\begin{aligned}
S_{m}^{2}= & \sum_{k \geqslant m}\left(\mathcal{C}(k, m)\left(I-P_{m}^{2}\right)\right)^{*} \mathcal{C}(k, m)\left(I-P_{m}^{2}\right) e^{2\left(\lambda^{\prime}-\rho^{\prime}\right)(k-m)} \\
& -\sum_{k<m}\left(\mathcal{C}(k, m) P_{m}^{2}\right)^{*} \mathcal{C}(k, m) P_{m}^{2} e^{2\left(\lambda^{\prime}-\rho^{\prime}\right)(m-k)}
\end{aligned}
$$

Using (3.20) and (3.21), one can now repeat previous arguments (working with $C_{m}$ instead of $B_{m}$ and $S_{m}^{2}$ instead of $S_{m}^{1}$ ) and show that (3.2), (3.3) and (3.4) hold for $i=2$ too. This completes the proof of the theorem.

We now establish the converse of Theorem 3.1.

Theorem 3.5. Let $\left(A_{m}\right)_{m \in \mathbb{Z}} \subset B(X)$ be a sequence of operators such that (3.1) holds with some $C>0$. Fur-

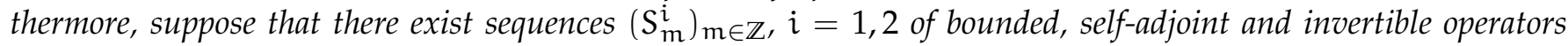
on $X$, sequences $\left(H_{m}^{i}\right)_{m \in \mathbb{Z}},\left(\tilde{H}_{m}^{i}\right)_{m \in \mathbb{Z}, i=1,2 \text { of self-adjoint operators } i n} B(X)$ and constants $K, \delta, \omega_{1}>0$ and $\omega_{2}<0$ such that (3.2), (3.3) and (3.4) hold for each $\mathrm{m} \in \mathbb{Z}$ and $i \in\{1,2\}$. Then, the sequence $\left(A_{m}\right)_{m \in \mathbb{Z}}$ admits an exponential trichotomy.

Proof. We define a sequence of operators $\left(B_{m}\right)_{m \in \mathbb{Z}}$ by $B_{m}=e^{\omega_{1}} A_{m}, m \in \mathbb{Z}$. Furthermore, let $T$ and $W$ be defined as in (3.13) and (3.14). It follows from (3.1) and (3.2) that $T$ and $W$ are well-defined. Moreover, the second inequality in (3.2) (for $i=1$ ) implies that $W$ is invertible and that the inverse is given by

$$
\left(W^{-1} \mathbf{x}\right)_{n}=\left(S_{n}^{1}\right)^{-1} x_{n}, \quad n \in \mathbb{Z}, \quad \mathbf{x}=\left(x_{n}\right)_{n \in \mathbb{Z}} \in l^{2} .
$$

We note that (3.3) and (3.4) imply that

$$
\mathrm{T}^{*} \mathrm{WT}-\mathrm{W}=-\mathrm{H}, \quad \text { and } \quad \mathrm{TW}^{-1} \mathrm{~T}^{*}-\mathrm{W}^{-1}=-\tilde{\mathrm{H}},
$$


where

$$
(H \mathbf{x})_{m}=H_{m}^{1} x_{m}, \quad \text { and }(\tilde{H} \mathbf{x})_{m}=\tilde{H}_{m}^{1} x_{m}, \quad \text { for } x=\left(x_{m}\right)_{m \in \mathbb{Z}} \in l^{2} .
$$

By (3.3) and (3.4), $H \geqslant \delta I$ and $\tilde{H} \geqslant \delta I$ and thus it follows from (3.22) and Theorem 2.2 that $T$ is hyperbolic. In particular, operator $I-T$ is invertible on $l^{2}$ and by Theorem 2.4 , the sequence $\left(B_{m}\right)_{m \in \mathbb{Z}}$ admits an exponential dichotomy. Similarly, one can show that sequence $\left(C_{m}\right)_{m \in \mathbb{Z}}$ defined by $C_{m}=e^{\omega_{2}} A_{m}$ admits an exponential dichotomy. Hence, there exist projections $\mathrm{P}_{\mathrm{m}}^{1}$ and $\mathrm{P}_{\mathrm{m}}^{2}$ for $\mathrm{m} \in \mathbb{Z}$ satisfying

$$
B_{m} P_{m}^{1}=P_{m+1}^{1} B_{m}, \quad C_{m} P_{m}^{2}=P_{m+1}^{2} C_{m},
$$

such that operators

$$
\mathrm{B}_{\mathrm{m}} \mid \operatorname{Ker} \mathrm{P}_{\mathrm{m}}^{1}: \operatorname{Ker} \mathrm{P}_{\mathrm{m}}^{1} \rightarrow \operatorname{Ker} \mathrm{P}_{\mathrm{m}+1}^{1}, \quad \text { and } \quad \mathrm{C}_{\mathrm{m}} \mid \operatorname{Ker} \mathrm{P}_{\mathrm{m}}^{2}: \operatorname{Ker} \mathrm{P}_{\mathrm{m}}^{2} \rightarrow \operatorname{Ker} \mathrm{P}_{\mathrm{m}+1}^{2},
$$

are invertible for $m \in \mathbb{Z}$ and there exist constants $\lambda, D>0$ such that

$$
\begin{gathered}
\left\|\mathcal{B}(m, n) P_{n}^{1}\right\| \leqslant D e^{-\lambda(m-n),} \\
\left\|\mathcal{C}(m, n) P_{n}^{2}\right\| \leqslant D e^{-\lambda(m-n),}
\end{gathered}
$$

for $m \geqslant n$ and

$$
\begin{gathered}
\left\|\mathcal{B}(m, n) Q_{n}^{1}\right\| \leqslant D e^{-\lambda(n-m)}, \\
\left\|\mathcal{C}(m, n) Q_{n}^{2}\right\| \leqslant D e^{-\lambda(n-m)},
\end{gathered}
$$

for $m \leqslant n$, where $Q_{n}^{i}=I-P_{n}^{i}$.

Lemma 3.6. For each $\mathrm{n} \in \mathbb{Z}$, we have

$$
\operatorname{Im} P_{n}^{1} \subset \operatorname{Im} P_{n}^{2}, \quad \text { and } \operatorname{Im} Q_{n}^{2} \subset \operatorname{Im} Q_{n}^{1} .
$$

Proof. Take $x \in \operatorname{Im} \mathrm{P}_{n}^{1}$. It follows from Proposition 2.3 that

$$
\sup _{m \geqslant n}\|\mathcal{B}(m, n) x\|<+\infty .
$$

Furthermore, we have

$$
\|\mathcal{C}(m, n) x\|=e^{\omega_{2}(m-n)}\|\mathcal{A}(m, n) x\|=e^{\left(\omega_{2}-\omega_{1}\right)(m-n)}\|\mathcal{B}(m, n) x\|,
$$

for $m \geqslant n$. Since $\omega_{2}-\omega_{1}<0$, we have that

$$
\sup _{m \geqslant n}\|\mathfrak{C}(m, n) x\|<+\infty .
$$

Using Proposition 2.3 we conclude that $x \in \operatorname{Im~}_{n}^{2}$. The proof of the second inclusion in (3.27) is analogous.

Lemma 3.7. The map $\mathrm{I}-\mathrm{P}_{\mathrm{n}}^{1}-\mathrm{Q}_{\mathrm{n}}^{2}$ is a projection for each $\mathrm{n} \in \mathbb{Z}$.

Proof. It follows from the previous lemma that

$$
\mathrm{P}_{\mathrm{n}}^{1} \mathrm{Q}_{\mathrm{n}}^{2}=\mathrm{Q}_{\mathrm{n}}^{2} \mathrm{P}_{\mathrm{n}}^{1}=0
$$

for $n \in \mathbb{Z}$. Hence

$$
\begin{aligned}
\left(I-P_{n}^{1}-Q_{n}^{2}\right)^{2} & =I-2 P_{n}^{1}-2 Q_{n}^{2}+\left(P_{n}^{1}\right)^{2}+\left(Q_{n}^{2}\right)^{2}+P_{n}^{1} Q_{n}^{2}+Q_{n}^{2} P_{n}^{1} \\
& =I-P_{n}^{1}-Q_{n}^{2}
\end{aligned}
$$

and the conclusion in the lemma follows. 
Lemma 3.8. For each $\mathrm{n} \in \mathbb{Z}$, we have

$$
\operatorname{Im}\left(I-P_{n}^{1}-Q_{n}^{2}\right)=\operatorname{Im} P_{n}^{2} \cap \operatorname{Im} Q_{n}^{1} .
$$

Proof. Take $x \in \operatorname{Im} P_{n}^{2} \cap \operatorname{Im} Q_{n}^{1}$. We have $Q_{n}^{2} x=P_{n}^{1} x=0$ and thus,

$$
\left(\mathrm{I}-\mathrm{P}_{\mathrm{n}}^{1}-\mathrm{Q}_{\mathrm{n}}^{2}\right) x=\chi .
$$

This implies that $x \in \operatorname{Im}\left(I-P_{n}^{1}-Q_{n}^{2}\right)$. Now take $x \in \operatorname{Im}\left(I-P_{n}^{1}-Q_{n}^{2}\right)$. We have $P_{n}^{1} x=-Q_{n}^{2} x$. Applying $P_{n}^{1}$, we obtain $P_{n}^{1} x=0$ and thus $x \in \operatorname{Im} Q_{n}^{1}$. Similarly, $x \in \operatorname{Im} P_{n}^{2}$ and so $x \in \operatorname{Im} P_{n}^{2} \cap \operatorname{Im} Q_{n}^{1}$.

We proceed with the proof of the theorem. By Lemma 3.8, the cocycle $\mathcal{A}(\mathrm{m}, \mathrm{n})$ is invertible along the ranges of projections $I d-P_{n}^{1}-Q_{n}^{2}$. It follows from (3.23) that

$$
\left\|\mathcal{A}(\mathrm{m}, \mathrm{n}) \mathrm{P}_{\mathrm{n}}^{1}\right\| \leqslant \mathrm{D} e^{-\left(\lambda+\omega_{1}\right)(m-n)}, \quad \text { for } \mathrm{m} \geqslant \mathrm{n} .
$$

Similarly, by (3.26) we have

$$
\left\|\mathcal{A}(\mathrm{m}, \mathrm{n}) \mathrm{Q}_{\mathrm{n}}^{2}\right\| \leqslant D D^{\prime} \mathrm{e}^{-\left(\lambda-\omega_{2}\right)(\mathrm{n}-\mathrm{m})}, \text { for } \mathrm{m} \leqslant \mathrm{n} .
$$

Moreover, it follows from (3.24), (3.25) and Lemma 3.8 that for each $x \in \operatorname{Im}\left(\operatorname{Id}-P_{n}^{1}-Q_{n}^{2}\right)$, we have

$$
\|\mathcal{A}(\mathrm{m}, \mathrm{n}) x\| \leqslant \mathrm{D} e^{-\left(\lambda+\omega_{2}\right)(\mathrm{m}-\mathrm{n})},
$$

for $m \geqslant n$ and

$$
\|\mathcal{A}(\mathrm{m}, \mathrm{n}) x\| \leqslant \mathrm{D} e^{-\left(\lambda-\omega_{1}\right)(\mathrm{n}-\mathrm{m})},
$$

for $m \leqslant n$. In addition, by (3.23) and (3.26) (applied for $m=n$ ),

$$
\left\|I-P_{n}^{1}-Q_{n}^{2}\right\| \leqslant 3 D,
$$

for $n \in \mathbb{Z}$. Hence,

$$
\left\|\mathcal{A}(m, n)\left(I-P_{n}^{1}-Q_{n}^{2}\right)\right\| \leqslant 3 D^{2} e^{-\left(\lambda+\omega_{2}\right)(m-n)}, \quad \text { for } m \geqslant n
$$

and

$$
\left\|\mathcal{A}(\mathrm{m}, \mathrm{n})\left(\mathrm{I}-\mathrm{P}_{\mathrm{n}}^{1}-\mathrm{Q}_{\mathrm{n}}^{2}\right)\right\| \leqslant 3 \mathrm{D}^{2} e^{-\left(\lambda-\omega_{1}\right)(\mathrm{n}-\mathrm{m})}, \quad \text { for } \mathrm{m} \leqslant \mathrm{n} .
$$

It follows from (3.28), (3.29), (3.30) and (3.31) that the sequence $\left(A_{m}\right)_{m \in \mathbb{Z}}$ admits an exponential trichotomy.

\section{Stability of trichotomies under linear perturbations}

In this section we use the results obtained in the previous section to establish in a simple manner the stability of exponential trichotomies under sufficiently small linear perturbations.

Theorem 4.1. Let $\left(A_{m}\right)_{m \in \mathbb{Z}}$ and $\left(B_{m}\right)_{m \in \mathbb{Z}}$ be sequences of bounded linear operators on $X$ such that:

1. the sequence $\left(A_{m}\right)_{m \in \mathbb{Z}}$ admits an exponential trichotomy and there exists $C>0$ such that (3.1) holds;

2. there exists $\rho>0$ such that

$$
\left\|A_{m}-B_{m}\right\| \leqslant \rho, \quad \text { for } m \in \mathbb{Z} .
$$

If $\rho$ is sufficiently small, then the sequence $\left(B_{m}\right)_{m \in \mathbb{Z}}$ admits an exponential trichotomy.

Proof. We first note that it follows from (3.1) and (4.1) that $\left\|B_{m}\right\| \leqslant C+\rho$, for all $m \in \mathbb{Z}$. By Theorem 3.1, there exist sequences $\left(S_{m}^{i}\right)_{m}, i=1,2$ of bounded, self-adjoint and invertible operators, sequences 
$\left(H_{m}^{i}\right)_{m \in \mathbb{Z}},\left(\tilde{H}_{m}^{i}\right)_{m \in \mathbb{Z}}, i=1,2$ of self-adjoint operators in $B(X)$ and constants $D, \delta, \omega_{1}>0$ and $\omega_{2}<0$ such that (3.2), (3.3) and (3.4) hold. Furthermore, we have

$$
\begin{aligned}
\left\langle e^{2 \omega_{i}} S_{m+1}^{i} B_{m} x, B_{m} x\right\rangle-\left\langle S_{m}^{i} x, x\right\rangle= & \left\langle e^{2 \omega_{i}} S_{m+1}^{i}\left(B_{m}-A_{m}\right) x,\left(B_{m}-A_{m}\right) x\right\rangle \\
& +\left\langle e^{2 \omega_{i}} S_{m+1}^{i} A_{m} x,\left(B_{m}-A_{m}\right) x\right\rangle \\
& +\left\langle e^{2 \omega_{i}} S_{m+1}^{i}\left(B_{m}-A_{m}\right) x, A_{m} x\right\rangle \\
& +\left\langle e^{2 \omega_{i}} S_{m+1}^{i} A_{m} x, A_{m} x\right\rangle-\left\langle S_{m}^{i} x, x\right\rangle,
\end{aligned}
$$

for each $x \in X, m \in \mathbb{Z}$ and $i=1,2$. On the other hand, it follows from (3.2) and (4.1) that

$$
\begin{aligned}
\left\langle e^{2 \omega_{i}} S_{m+1}^{i}\left(B_{m}-A_{m}\right) x,\left(B_{m}-A_{m}\right) x\right\rangle & \leqslant e^{2 \omega_{1}}\left\|S_{m+1}^{i}\left(B_{m}-A_{m}\right) x\right\| \cdot\left\|\left(B_{m}-A_{m}\right) x\right\| \\
& \leqslant K \rho^{2} e^{2 \omega_{1}}\langle x, x\rangle .
\end{aligned}
$$

Similarly, by (3.1), (3.2) and (4.1), we have

$$
\left\langle e^{2 \omega_{i}} S_{m+1}^{i} A_{m} x,\left(B_{m}-A_{m}\right) x\right\rangle \leqslant K C e^{2 \omega_{1}} \rho\langle x, x\rangle,
$$

and

$$
\left\langle e^{2 \omega_{i}} S_{m+1}^{i}\left(B_{m}-A_{m}\right) x, A_{m} x\right\rangle \leqslant K C e^{2 \omega_{1}} \rho\langle x, x\rangle .
$$

Hence, it follows from (3.3) that

$$
\left\langle e^{2 \omega_{i}} S_{m+1}^{i} B_{m} x, B_{m} x\right\rangle-\left\langle S_{m}^{i} x, x\right\rangle \leqslant\left(K \rho^{2} e^{2 \omega_{1}}+2 K C \rho e^{2 \omega_{1}}-\delta\right)\langle x, x\rangle,
$$

for each $m \in \mathbb{Z}$ and $x \in X$. Setting $r=-K \rho^{2} e^{2 \omega_{1}}-2 K C \rho e^{2 \omega_{1}}+\delta$, we note that for $\rho$ sufficiently small, we have that $r>0$ and

$$
e^{2 \omega_{i}} B_{m}^{*} S_{m+1}^{i} B_{m}-S_{m}^{i}=-W_{m}, \quad W_{m} \geqslant r I, \quad \text { for } m \in \mathbb{Z} \text { and } i=1,2 .
$$

Similarly, one can show that

$$
e^{2 \omega_{i}} B_{m}\left(S_{m}^{i}\right)^{-1} B_{m}^{*}-\left(S_{m+1}^{i}\right)^{-1}=-\tilde{W}_{m}, \quad \tilde{W}_{m} \geqslant r I, \quad \text { for } m \in \mathbb{Z} \quad \text { and } i=1,2 .
$$

By (3.2), (4.2), (4.3) and Theorem 3.5, the sequence $\left(B_{m}\right)_{m \in \mathbb{Z}}$ admits an exponential trichotomy. The proof of the theorem is completed.

We note that the version of Theorem 4.1 is valid when $X$ is an arbitrary Banach space and without the assumption that (3.1) holds (see [3, 12]). However, we are not able to establish this general version using our methods.

\section{Lyapunov sequences and nonlinear perturbations}

We consider the nonlinear dynamics

$$
x_{m+1}=A_{m} x_{m}+f_{m}\left(x_{m}\right),
$$

where $f_{m}: X \rightarrow X, m \in \mathbb{Z}$ are continuous functions. We are going to show that if the linear part of the equation (5.1) admits an exponential trichotomy and if the nonlinear perturbation is sufficiently small that then each solution of (5.1) has the property that the associated sequence obtained by projecting the solution on the stable subspace of our trichotomy is uniformly exponentially stable. The precise statement is given below.

Theorem 5.1. Assume that the sequence $\left(A_{m}\right)_{m \in \mathbb{Z}}$ admits an exponential trichotomy and that the sequence $\left(f_{m}\right)_{m \in \mathbb{Z}}$ satisfies: 
1. there exists $\rho>0$ such that

$$
\left\|\mathrm{f}_{\mathrm{m}}(\mathrm{x})\right\| \leqslant \rho\|x\|, \quad \text { for } \mathrm{m} \in \mathbb{Z} \text { and } \mathrm{x} \in \mathrm{X}
$$

2.

$$
P_{m+1}^{1} f_{m}(x)=f_{m}\left(P_{m}^{1} x\right), \quad \text { for } m \in \mathbb{Z} \text { and } x \in X .
$$

Then for sufficiently small $\rho$, there exists $\mathrm{L}>0$ and $\eta \in(0,1)$ such that

$$
\left\|P_{n}^{1} x_{n}\right\| \leqslant L \eta^{n-m}\left\|P_{m}^{1} x_{m}\right\|
$$

for $m \geqslant n$ and every solution $\left(x_{m}\right)_{m \in \mathbb{Z}}$ of (5.1).

Proof. Consider operators $S_{m}^{1}, m \in \mathbb{Z}$ defined as in (3.11). We define a sequence of functions $H_{m}, m \in \mathbb{Z}$ by

$$
H_{m}(x)=\left\langle S_{m}^{1} x, x\right\rangle, \quad x \in X .
$$

Furthermore, let $u_{m}=P_{m}^{1} x_{m}, m \in \mathbb{Z}$. In the proof of Theorem 3.1 we have showed that (recalling that $\left.B_{m}=e^{\omega_{1}} A_{m}\right)$

$$
\begin{aligned}
B_{m}^{*} S_{m+1}^{1} B_{m}= & e^{-2(\lambda-\rho)} \sum_{k \geqslant m}\left(\mathcal{B}(k, m) P_{m}^{1}\right)^{*} \mathcal{B}(k, m) P_{m}^{1} e^{2(\lambda-\rho)(k-m)}-e^{-2(\lambda-\rho)}\left(P_{m}^{1}\right)^{*} P_{m}^{1} \\
& -e^{2(\lambda-\rho)} \sum_{k<m}\left(\mathcal{B}(k, m)\left(I-P_{m}^{1}\right)\right)^{*} \mathcal{B}(k, m)\left(I-P_{m}^{1}\right) e^{2(\lambda-\rho)(m-k)} \\
& -e^{2(\lambda-\rho)}\left(I-P_{m}^{1}\right)^{*}\left(I-P_{m}^{1}\right) .
\end{aligned}
$$

In particular, this implies that

$$
e^{2 \omega_{1}} A_{m}^{*} S_{m+1}^{1} A_{m} \leqslant e^{-2(\lambda-\rho)} S_{m}^{1}, \quad \forall m \in \mathbb{Z} .
$$

Using (5.3), we have

$$
\begin{aligned}
\mathrm{H}_{m+1}\left(u_{m+1}\right)= & \left\langle S_{m+1}^{1} u_{m+1}, u_{m+1}\right\rangle \\
= & \left\langle S_{m+1}^{1} P_{m+1}^{1} x_{m+1}, P_{m+1}^{1} x_{m+1}\right\rangle \\
= & \left\langle S_{m+1}^{1} P_{m+1}^{1}\left(A_{m} x_{m}+f_{m}\left(x_{m}\right)\right), P_{m+1}^{1}\left(A_{m} x_{m}+f_{m}\left(x_{m}\right)\right\rangle\right. \\
= & \left\langle S_{m+1}^{1}\left(A_{m} u_{m}+f_{m}\left(u_{m}\right)\right), A_{m} u_{m}+f_{m}\left(u_{m}\right)\right\rangle \\
= & \left\langle A_{m}^{*} S_{m+1}^{1} A_{m} u_{m}, u_{m}\right\rangle+\left\langle S_{m+1}^{1} A_{m} u_{m}, f_{m}\left(u_{m}\right)\right\rangle \\
& +\left\langle S_{m+1}^{1} f_{m}\left(u_{m}\right), A_{m} u_{m}\right\rangle+\left\langle S_{m+1}^{1} f_{m}\left(u_{m}\right), f_{m}\left(u_{m}\right)\right\rangle .
\end{aligned}
$$

By (3.1), (3.2), (5.2) and (5.5),

$$
\begin{aligned}
& \mathrm{H}_{\mathrm{m}+1}\left(\mathrm{u}_{\mathrm{m}+1}\right) \leqslant e^{-2(\lambda-\rho)-2 \omega_{1}} \mathrm{H}_{\mathfrak{m}}\left(\mathrm{u}_{\mathrm{m}}\right)+2\left\|S_{\mathrm{m}+1}^{1}\right\| \cdot\left\|A_{\mathfrak{m}} u_{m}\right\| \cdot\left\|f_{m}\left(u_{m}\right)\right\| \\
& +\left\|S_{m+1}^{1}\right\| \cdot\left\|f_{m}\left(u_{m}\right)\right\|^{2} \\
& \leqslant e^{-2(\lambda-\rho)-2 \omega_{1}} \mathrm{H}_{m}\left(u_{m}\right)+2 K C \rho\left\|u_{m}\right\|^{2}+K \rho^{2}\left\|u_{m}\right\|^{2} .
\end{aligned}
$$

Noting that $\mathrm{H}_{\mathrm{n}}(z) \geqslant\|z\|^{2}$ for $z \in \operatorname{Im}_{\mathrm{n}}^{1}$ and that $\mathrm{u}_{\mathrm{n}} \in \operatorname{Im} \mathrm{P}_{\mathfrak{n}}^{1}$, we conclude that

$$
H_{m+1}\left(u_{m+1}\right) \leqslant \eta^{2} H_{m}\left(u_{m}\right),
$$

where

$$
\eta^{2}=e^{-2(\lambda-\rho)-2 \omega_{1}}+2 K C \rho+K \rho^{2}
$$

By choosing $\rho$ sufficiently small, we can achieve that $\eta \in(0,1)$. Iterating (5.6), we obtain that

$$
H_{n}\left(u_{n}\right) \leqslant \eta^{2(n-m)} H_{m}\left(u_{m}\right), \quad \text { for } n \geqslant m .
$$

Since $\left\|u_{n}\right\|^{2} \leqslant H_{n}\left(u_{n}\right)$ and $H_{m}\left(u_{m}\right) \leqslant K\left\|u_{m}\right\|^{2}$, we conclude that (5.4) holds with $L=\sqrt{K}$. 


\section{Characterization of exponential trichotomies on $\mathbb{Z}_{0}^{+}$}

In this section we establish versions of the results of Section 3 for trichotomies on the half-line $\mathbb{Z}_{0}^{+}$. For each $n \geqslant 0$, let

$$
\mathrm{S}(\mathrm{n})=\left\{v \in X: \sup _{\mathrm{m} \geqslant \mathrm{n}}\|\mathcal{A}(\mathrm{m}, \mathrm{n}) v\|<+\infty\right\}
$$

We begin with the following version of Proposition 2.3 for dichotomies on $\mathbb{Z}_{0}^{+}$. It turns out that in this case only the ranges of projections $P_{n}^{1}$ are uniquely determined (see [45]).

Proposition 6.1. We have $\operatorname{Im} \mathrm{P}_{n}^{1}=\mathrm{S}(\mathrm{n})$, for each $\mathrm{n} \geqslant 0$.

We are also able to say what happens with the ranges of projections $\mathrm{P}_{\mathrm{n}}^{2}$ (again we refer to [45]).

Proposition 6.2. Assume that the sequence $\left(A_{m}\right)_{m \geqslant 0}$ of bounded operators on $X$ admits an exponential dichotomy and let $Z$ be any closed subspace of $X$ such that $X=S \oplus Z$. Then, $\left(A_{m}\right)_{m \geqslant 0}$ admits an exponential dichotomy with respect to projections $\mathrm{P}_{\mathrm{m}}^{\mathrm{i}}, \mathrm{m} \geqslant 0, i=1,2$ such that $\operatorname{Im} \mathrm{P}_{\mathrm{m}}^{2}=\mathcal{A}(\mathrm{m}, 0) \mathrm{Z}$.

In other words, the unstable direction at time $m \geqslant 0$ can be an image under the action of the cocycle of any closed subspace $Z$ with the property that $X$ can be decomposed as a direct sum of $S(0)$ and $Z$.

We can now comment on our strategy of establishing versions of results from Section 3 for trichotomies on the half-line $\mathbb{Z}_{0}^{+}$. We first emphasize that one is not able to proceed using the same type of arguments as in the proofs of Theorems 3.1 and 3.5. The major reason for this is the fact that Theorem 2.4 is no longer valid when one passes to the case of dichotomies on the half-line and consequently dichotomies on $\mathbb{Z}_{0}^{+}$cannot be characterized in terms of the invertibility (hyperbolicity) of a single operator. For appropriate versions of Theorem 2.4 for dichotomies on the half-line we refer to [45]. In order to overcome this difficulty, we will apply a different strategy of extending dichotomies on $\mathbb{Z}_{0}^{+}$to dichotomies on $\mathbb{Z}$ and applying already established results. The following result which is only a particular case of result established in [4] will be crucial.

Theorem 6.3. A sequence $\left(A_{m}\right)_{m \in \mathbb{Z}} \subset \mathrm{B}(\mathrm{X})$ admits an exponential dichotomy on $\mathbb{Z}$, if and only if there exist projections $\mathrm{P}_{\mathrm{m}}^{+}$for $\mathrm{m} \geqslant 0$ and projections $\mathrm{P}_{\mathrm{m}}^{-}$for $\mathrm{m} \leqslant 0$ such that:

1. $\left(A_{m}\right)_{m \geqslant 0}$ admits an exponential dichotomy on $\mathbb{Z}_{0}^{+}$with projections $\mathrm{P}_{\mathrm{m}}^{+}$;

2. $\left(A_{m}\right)_{m \leqslant 0}$ admits an exponential dichotomy on $\mathbb{Z}_{0}^{-}$with projections $\mathrm{P}_{\mathrm{m}}^{-}$;

3. $\mathrm{X}=\operatorname{Im} \mathrm{P}_{0}^{+} \oplus \operatorname{Ker} \mathrm{P}_{0}^{-}$.

The following is a version of Theorem 3.1 for dichotomies on $\mathbb{Z}_{0}^{+}$.

Theorem 6.4. Assume that the sequence $\left(A_{m}\right)_{m \in \mathbb{Z}_{0}^{+}} \subset B(X)$ admits an exponential trichotomy and that there exists $\mathrm{C}>0$ such that

$$
\left\|A_{m}\right\| \leqslant C, \quad \text { for } m \geqslant 0 .
$$

Then, there exist sequences $\left(S_{m}^{i}\right)_{m \geqslant 0}, i=1,2$ of bounded, self-adjoint and invertible operators on $X$, constants $K, \delta, \omega_{1}>0$ and $\omega_{2}<0$ such that (3.2), (3.3) and (3.4) hold for each $m \geqslant 0$ and $i \in\{1,2\}$.

Proof. As in the proof of Theorem 3.1, take an arbitrary $\omega_{1} \in(c, d)$ and consider the sequence $B_{m}=$ $e^{\omega_{1}} A_{m}, m \geqslant 0$. It follows from (3.9) and (3.10) that the sequence $\left(B_{m}\right)_{m \geqslant 0}$ admits an exponential dichotomy with projections $\mathrm{P}_{\mathrm{m}}^{+}=\mathrm{P}_{\mathrm{m}}^{1}$. Choose an arbitrary hyperbolic operator $\mathrm{B} \in \mathrm{B}(\mathrm{X})$ such that both $\operatorname{Im} \mathrm{P}_{0}^{+}$and $\mathrm{Ker}_{0}^{+}$are invariant under $\mathrm{B}$ and with the property that the spectrum of restriction $\left.\mathrm{B}\right|_{\operatorname{Im} \mathrm{P}_{0}^{+}}$ is contained in $\{\lambda:|\lambda|<1\}$ while the spectrum of restriction $\left.B\right|_{\operatorname{Ker} P_{0}^{+}}$is contained in $\{\lambda:|\lambda|>1\}$. Furthermore, extend the sequence $\left(B_{m}\right)_{m \geqslant 0}$ to a sequence defined on the whole line by setting $B_{m}=B$ for $m<0$. It follows from Theorem 6.3 that the sequence $\left(B_{m}\right)_{m \in \mathbb{Z}}$ admits an exponential dichotomy on $\mathbb{Z}$. One can now proceed as in the proof of Theorem 3.1 and construct a sequence $\left(S_{m}^{1}\right)_{m \in \mathbb{Z}}$ of bounded, self-adjoint and invertible operators on $X$ satisfying (3.2), (3.3) and (3.4) for $i=1$ and $m \in \mathbb{Z}$. In particular, 
those inequalities hold for $m \geqslant 0$ which establishes the assertion of the theorem for $i=1$. Similarly, one constructs the sequence $\left(S_{m}^{2}\right)_{m \geqslant 0}$.

The following is a converse of Theorem 6.4.

Theorem 6.5. Let $\left(A_{m}\right)_{m \geqslant 0} \subset B(X)$ be a sequence of operators such that (6.1) holds with some $C>0$. Furthermore, suppose that there exist sequences $\left(S_{m}^{i}\right)_{m \geqslant 0}, i=1,2$ of bounded, self-adjoint and invertible operators on $X$, constants $K, \delta, \omega_{1}>0$ and $\omega_{2}<0$ such that the inequalities (3.2), (3.3) and (3.4) hold for each $\mathrm{m} \geqslant 0$ and $i \in\{1,2\}$. Then the sequence $\left(A_{m}\right)_{m \geqslant 0}$ admits an exponential trichotomy.

Proof. It is an easy exercise in the functional calculus for self-adjoint operators to show that there exists $A \in B(X)$ self-adjoint such that

$$
e^{2 \omega_{1}} A S_{0}^{1} A-S_{0}^{1} \leqslant-\delta I
$$

By Theorem 2.2, we also have that

$$
\mathrm{e}^{2 \omega_{1}} \mathrm{AS} \mathrm{S}_{0}^{1} \mathrm{~A}-\mathrm{S}_{0}^{1} \leqslant-\delta \mathrm{I}
$$

Setting $A_{n}=A$ and $S_{n}^{1}=S_{0}^{1}$ for $n<0$, we conclude that for $i=1$, the inequalities (3.2), (3.3) and (3.4) hold for every $m \in \mathbb{Z}$. Arguing as in the proof of Theorem 3.5, one concludes that the sequence $\left(e^{\omega_{1}} A_{m}\right)_{m \in \mathbb{Z}}$ admits an exponential dichotomy on $\mathbb{Z}$ which readily implies that the sequence $\left(e^{\omega_{1}} A_{m}\right)_{m \geqslant 0}$ admits an exponential dichotomy on $\mathbb{Z}_{0}^{+}$with respect to projections $\mathrm{P}_{\mathrm{m}}^{1}$ and $\mathrm{Q}_{\mathrm{m}}^{1}=\mathrm{I}-\mathrm{P}_{\mathrm{m}}^{1}, \mathrm{~m} \geqslant 0$. Similarly, the sequence $\left(e^{\omega_{2}} A_{m}\right)_{m \geqslant 0}$ admits an exponential dichotomy on $\mathbb{Z}_{0}^{+}$with respect to projections $P_{m}^{2}$ and $\mathrm{Q}_{\mathrm{m}}^{2}=\mathrm{Id}-\mathrm{P}_{\mathrm{m}}^{2}, \mathrm{~m} \geqslant 0$ Using Proposition 6.1, one can argue as in the proof of Lemma 3.6 and obtain the first inclusion in (3.27). Moreover, it follows from Proposition 6.2 that we may assume that the second inclusion in (3.27) also holds. One can now proceed as in the proof of Theorem 3.5 and conclude that the sequence $\left(A_{m}\right)_{m} \geqslant 0$ admits an exponential trichotomy.

Using Theorems 6.4 and 6.5, one can now establish versions of Theorems 4.1 and 5.1 for trichotomies on $\mathbb{Z}_{0}^{+}$with the exact same proofs as in the case of trichotomies on $\mathbb{Z}$.

\section{Acknowledgment}

D. D. was supported by an Australian Research Council Discovery Project DP150100017 and by the Croatian Science Foundation under the project IP-2014-09-2285. D. D. would also like to thank the referees for valuable comments.

\section{References}

[1] A. I. Alonso, J.-L. Hong, R. Obaya, Exponential dichotomy and trichotomy for difference equations, Comput. Math. Appl., 38 (1999), 41-49. 1

[2] L. Barreira, D. Dragičević, C. Valls, Exponential dichotomies with respect to a sequence of norms and admissibility, Internat. J. Math., 23 (2014), 20 pages. 2.2

[3] L. Barreira, D. Dragičević, C. Valls, Admissibility and nonuniform exponential trichotomies, Regul. Chaotic Dyn., 20 (2015), 49-62. 1, 4

[4] L. Barreira, D. Dragičević, C. Valls, From one-sided dichotomies to two-sided dichotomies, Discrete Contin. Dyn. Syst., 35 (2015), 2817-2844. 6

[5] L. Barreira, D. Dragičević, C. Valls, Characterization of nonuniform exponential trichotomies for flows, J. Math. Anal. Appl., 434 (2016), 376-400. 1

[6] L. Barreira, D. Dragičević, C. Valls, Nonuniform exponential dichotomies and Lyapunov functions, Regul. Chaotic Dyn., to appear. 1

[7] L. Barreira, C. Valls, Center manifolds for nonuniformly partially hyperbolic diffeomorphisms, J. Math. Pures Appl., 84 (2005), 1693-1715. 1

[8] L. Barreira, C. Valls, Smooth center manifolds for nonuniformly partially hyperbolic trajectories, J. Differential Equations, 237 (2007), 307-342. 1

[9] L. Barreira, C. Valls, Growth rates and nonuniform hyperbolicity, Discrete Contin. Dyn. Syst., 22 (2008), 509-528. 1 
[10] L. Barreira, C. Valls, Stability of nonautonomous differential equations, Lecture Notes in Mathematics, Springer, Berlin, (2008). 1

[11] L. Barreira, C. Valls, Lyapunov sequences for exponential trichotomies, Nonlinear Anal., 72 (2010), 192-203. 1

[12] L. Barreira, C. Valls, Robustness of dichotomies and trichotomies for difference equations, Port. Math., 71 (2014), 277-300. 1,4

[13]

[14] M. I. Brin, J. B. Pesin, Partially hyperbolic dynamical systems, (Russian) Izv. Akad. Nauk SSSR Ser. Mat., 38 (1974), 177-212. 1

[15] J. Carr, Applications of centre manifold theory, Applied Mathematical Sciences, Springer-Verlag, New York-Berlin, (1981). 1

[16] C. Chicone, Y. Latushkin, Center manifolds for infinite-dimensional nonautonomous differential equations, J. Differential Equations, 141 (1997), 356-399. 1

[17] S.-N. Chow, W.-S. Liu, Y.-F. Yi, Center manifolds for invariant sets, Special issue in celebration of Jack K. Hale's 70th birthday, Part 2, Atlanta, GA/Lisbon, (1998), J. Differential Equations, 168 (2000), 355-385.

[18] S.-N. Chow, W.-S. Liu, Y.-F. Yi, Center manifolds for smooth invariant manifolds, Trans. Amer. Math. Soc., 352 (2000), 5179-5211.

[19] S.-N. Chow, Y.-F. Yi, Center manifold and stability for skew-product flows, J. Dynam. Differential Equations, 6 (1994), 543-582. 1

[20] J. L. Daleckiř, M. G. Krel̆n, Stability of solutions of differential equations in Banach space, Translated from the Russian by S. Smith, Translations of Mathematical Monographs, American Mathematical Society, Providence, R.I., (1974). $1,2.1$

[21] R. Datko, Extending a theorem of A. M. Liapunov to Hilbert space, J. Math. Anal. Appl., 32 (1970), 610-616. 1

[22] D. Dragičević, A version of a theorem of R. Datko for stability in average, Systems Control Lett., 96 (2016), 1-6. 1

[23] D. Dragičević, C. Preda, Lyapunov theorems for exponential dichotomies in Hilbert spaces, Internat. J. Math., 27 (2016), 13 pages. 1

[24] A. Ducrot, P. Magal, O. Seydi, Persistence of exponential trichotomy for linear operators: a Lyapunov-Perron approach, J. Dynam. Differential Equations, 28 (2016), 93-126. 1

[25] S. Elaydi, O. Hájek, Exponential trichotomy of differential systems, J. Math. Anal. Appl., 129 (1988), 362-374. 1

[26] S. Elaydi, K. Janglajew, Dichotomy and trichotomy of difference equations, J. Differ. Equations Appl., 3 (1998), $417-448$. 1

[27] K. J. Engel, R. Nagel, One-parameter semigroups for linear evolution equations, With contributions by S. Brendle, M. Campiti, T. Hahn, G. Metafune, G. Nickel, D. Pallara, C. Perazzoli, A. Rhandi, S. Romanelli and R. Schnaubelt, Graduate Texts in Mathematics, Springer-Verlag, New York, (2000). 1

[28] M. Faieghi, S. K. Mousavi Mashhadi, D. Baleanu, Sampled-data nonlinear observer design for chaos synchronization: a Lyapunov-based approach, Commun. Nonlinear Sci. Numer. Simul., 19 (2014), 2444-2453. 1

[29] T. Gallay, A center-stable manifold theorem for differential equations in Banach spaces, Comm. Math. Phys., 152 (1993), 249-268. 1

[30] J. A. Goldstein, On the operator equation $A X+X B=Q$, Proc. Amer. Math. Soc., 70 (1978), 31-34. 1

[31] J. K. Hale, L. T. Magalhães, W. M. Oliva, Dynamics in infinite dimensions, With an appendix by Krzysztof P. Rybakowski, Second edition, Applied Mathematical Sciences, Springer-Verlag, New York, (2002). 1

[32] B. Hasselblatt, Y. Pesin, Partially hyperbolic dynamical systems, Handbook of dynamical systems, Elsevier B. V., Amsterdam, 1B (2005), 1-55. 1

[33] D. Henry, Geometric theory of semilinear parabolic equations, Lecture Notes in Mathematics, Springer-Verlag, BerlinNew York, (1981). 1

[34] Y.-X. Jiang, F.-F. Liao, Lyapunov functions for general nonuniform trichotomy with different growth rates, Electron. J. Differential Equations, 2014 (2014), 17 pages. 1

[35] J. López-Fenner, M. Pinto, ( $h, k)$-trichotomies and asymptotics of nonautonomous difference systems, Comput. Math. Appl., 33 (1997), 105-124. 1

[36] G. Papaschinopoulos, On exponential trichotomy of linear difference equations, Appl. Anal., 40 (1991), 89-109. 1

[37] A. Pazy, Semigroups of linear operators and applications to partial differential equations, Applied Mathematical Sciences, Springer-Verlag, New York, (1983). 1

[38] V. Q. Phóng, The operator equation $\mathrm{AX}-\mathrm{XB}=\mathrm{C}$ with unbounded operators $\mathrm{A}$ and $\mathrm{B}$ and related abstract Cauchy problems, Math. Z., 208 (1991), 567-588. 1

[39] A. Pogan, C. Preda, P. Preda, A discrete Lyapunov theorem for the exponential stability of evolution families, New York J. Math., 11 (2005), 457-463. 1

[40] C. Preda, P. Preda, F. Bătăran, An extension of a theorem of R. Datko to the case of (non)uniform exponential stability of linear skew-product semiflows, J. Math. Anal. Appl., 425 (2015), 1148-1154.

[41] C. Preda, P. Preda, A. Craciunescu, A version of a theorem of R. Datko for nonuniform exponential contractions, J. Math. Anal. Appl., 385 (2012), 572-581. 1

[42] A. L. Sasu, Exponential dichotomy and dichotomy radius for difference equations, J. Math. Anal. Appl., 344 (2008), 906-920.2.2 
[43] A. L. Sasu, B. Sasu, Input-output admissibility and exponential trichotomy of difference equations, J. Math. Anal. Appl., 380 (2011), 17-32. 1

[44] B. Sasu, A. L. Sasu, Nonlinear criteria for the existence of the exponential trichotomy in infinite dimensional spaces, Nonlinear Anal., 74 (2011), 5097-5110. 1

[45] B. Sasu, A. L. Sasu, On the dichotomic behavior of discrete dynamical systems on the half-line, Discrete Contin. Dyn. Syst., 33 (2013), 3057-3084. 6, 6, 6

[46] A. L. Sasu, B. Sasu, A Zabczyk type method for the study of the exponential trichotomy of discrete dynamical systems, Appl. Math. Comput., 245 (2014), 447-461. 1

[47] A. L. Sasu, B. Sasu, Discrete admissibility and exponential trichotomy of dynamical systems, Discrete Contin. Dyn. Syst., 34 (2014), 2929-2962.

[48] A. L. Sasu, B. Sasu, Admissibility and exponential trichotomy of dynamical systems described by skew-product flows, J. Differential Equations, 260 (2016), 1656-1689. 1

[49] G. R. Sell, Y.-C. You, Dynamics of evolutionary equations, Applied Mathematical Sciences, Springer-Verlag, New York, (2002). 1

[50] A. Vanderbauwhede, G. Iooss, Center manifold theory in infinite dimensions, Dynamics reported: expositions in dynamical systems, Dynam. Report. Expositions Dynam. Systems (N.S.), Springer, Berlin, 1 (1992), 125-163. 1

[51] A. Vanderbauwhede, S. A. van Gils, Center manifolds and contractions on a scale of Banach spaces, J. Funct. Anal., 72 (1987), 209-224. 1

[52] G.-C. Wu, D. Baleanu, Jacobian matrix algorithm for Lyapunov exponents of the discrete fractional maps, Commun. Nonlinear Sci. Numer. Simul., 22 (2015), 95-100. 1 